\title{
Neuroprotective Effect of Genistein in Peripheral Nerve Injury
}

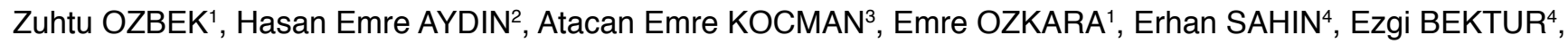 \\ Murat VURAL' ${ }^{1}$, Aydan $\mathrm{KOSE}^{3}$, Ali ARSLANTAS ${ }^{1}$, Cengiz BAYCU ${ }^{4}$ \\ ${ }^{1}$ Eskisehir Osmangazi University, Department of Neurosurgery, Eskisehir, Turkey \\ ${ }^{2}$ Dumlupınar University, Department of Neurosurgery, Kutahya, Turkey \\ ${ }^{3}$ Eskisehir Osmangazi University, Department of Plastic Surgery, Eskisehir, Turkey \\ ${ }^{4}$ Eskisehir Osmangazi University, Department of Histology, Eskisehir, Turkey
}

\section{ABSTRACT}

AIM: To investigate the effects of genistein in a rat model of sciatic nerve crush injury and complete sciatic nerve transection. The effects of genistein were compared with those of gabapentin, which is widely used in clinical practice for peripheral nerve injury.

MATERIAL and METHODS: Forty-eight rats were randomly divided into six groups (8 rats in each group): group 1 (sham); group 2, sciatic nerve crush injury (control); group 3, sciatic nerve crush injury+genistein $20 \mathrm{mg} / \mathrm{kg}$; group 4, sciatic nerve crush injury+gabapentin $90 \mathrm{mg} / \mathrm{kg}$; group 5, sciatic nerve transection+genistein $20 \mathrm{mg} / \mathrm{kg}$; group 6, sciatic nerve transection+gabapentin $90 \mathrm{mg} / \mathrm{kg}$. The effects of genistein and gabapentin were assessed with immunohistochemical staining for growth associated protein-43 (GAP-43) and myelin basic protein (MBP). Interleukin-1 $\beta$ and tumor necrosis factor a levels in the injured nerve specimens were assessed as a measure of inflammatory response; walking track analysis and sciatic function index for neurological recovery and the paw mechanical withdrawal threshold were examined for neuropathic pain.

RESULTS: On histopathological examination, genistein use was associated with a greater immunoreactivity for GAP-43 and MBP compared with that associated with gabapentin. Genistein and gabapentin had similar effects on anti-inflammatory activity, functional recovery, and neuropathic pain.

CONCLUSION: Genistein and gabapentin exhibit positive effects on histopathology, inflammation, and clinical findings of peripheral nerve injury. When the systemic side effects of gabapentin are considered, genistein (a basic soy isoflavone that has no side effects) can be used as an alternative to medical treatment in peripheral nerve injury.

KEYWORDS: Genistein, Gabapentin, Sciatic nerve injury, Neuroprotection

\section{INTRODUCTION}

A Ithough microsurgical techniques have been developed and positive effects of clinically and experimentally different neurotrophic drugs, steroids, hormones, and even low-dose radiation have been reported, desirable motor and sensory recovery after peripheral nerve injury is a clinical challenge $(6,16,18,20,25)$. Methylprednisolone and gabapentin are considered as reference agents, against which the medical treatment of traumatic peripheral nerve injury is evaluated. However, their adverse effects are a major limitation associated with their clinical use (16).
Genistein was shown to promote recovery in experimental peripheral neuropathy and chronic peripheral nerve injury $(23,24)$. However, the effect of genistein in animal models of acute crush injury or complete transection of peripheral nerve has not yet been investigated.

The purpose of this study was to investigate the effects of genistein after experimental sciatic nerve crush injury and complete sciatic nerve transection in rats and to compare its effects with those of gabapentin. 


\section{MATERIAL and METHODS}

The Medical and Surgical Research Center of Eskisehir Osmangazi University and Committee on Animal Experiments of the Medical Faculty approved the present experimental study (Permit No. 2014/384). All experimental procedures were performed in accordance with the National Institute of Health Principles of Laboratory Animal Care.

\section{Animal sample preparation and surgical procedure}

Forty-eight male Sprague-Dawley rats (200-250 g each) were divided into six groups of eight rats each. The rats were housed in separate cages during the test and were fed standard rodent food and water. They were fasted for 12 hours prior to the operation, but had ad libitum access to water. Paw mechanical withdrawal threshold, walking track analysis, and sciatic function index were measured between 9 and 15 hours of daylight.

Intraperitoneal sodium pentobarbital $(50 \mathrm{mg} / \mathrm{kg}$ ) was used to induce anesthesia during the surgical procedure in all groups. The right hind legs of the animals were de-epithelized with creams before the surgery, and the surgical field cleaned with povidone iodine solution. A skin incision was made on the right hip of the rats in the group 1 (sham) and the sciatic nerve within the gluteal muscle exposed. The surgical wound was closed without injury, and dimethyl sulfoxide (solvent, DMSO) was intraperitoneally administered. The same procedure was followed for rats in the group 2 (control) plus a force of 54 $\mathrm{N}$ was applied on the sciatic nerve for 60 seconds with an aneurysm clip placed $0.5 \mathrm{~cm}$ proximal to its trifurcation and the crush injury achieved. After the crush injury was inflicted in rats in group $3,20 \mathrm{mg} / \mathrm{kg}$ genistein was administered intraperitoneally for 30 days. Rats in group 4 underwent the same procedure as in group 3. After inflicting the crush injury, $90 \mathrm{mg} / \mathrm{kg}$ gabapentin was administered intraperitoneally for 30 days. The sciatic nerve was sectioned transversely through the full-thickness in rats in group 5 and 6 and the truncated portions sutured epineurally at four sites with $9 / 0$ nylon suture, aided by binocular loupes (Supervu loupes $2.5 \times 42 \mathrm{~cm}$ ). Subsequently, $20 \mathrm{mg} / \mathrm{kg}$ genistein and $90 \mathrm{mg} / \mathrm{kg}$ gabapentin was administered intraperitoneally for 30 days in the rats in groups 5 and 6, respectively.

Rats were sacrificed after the last walking track analysis and mechanical withdrawal threshold measurement at $4^{\text {th }}$ week. The right hind limb was re-exposed and the sciatic nerve harvested en-bloc after careful dissection. The removed sciatic nerve was sent to the laboratory for immunohistochemical and biochemical analyses.

\section{Immunohistochemical analysis}

Tissue samples from each experimental group were fixed in neutral formalin for 72 hours and embedded in paraffin. Sections of 4-5 $\mu \mathrm{m}$ thickness were processed for polylysine microscope slides. Heat-induced antigen retrieval was performed to increase the sensitivity of immunohistochemical examination. Endogenous peroxidase activity was blocked in 3\% hydrogen peroxide. Epitopes were stabilized by application of serumblocking solution. Slides were incubated overnight with myelin basic protein (MBP) antibody (C-16, Santa Cruz-13914) and growth-associated protein 43 (GAP-43) antibody (B-5, Santa Cruz-17790) at $4^{\circ} \mathrm{C}$. After incubation, the secondary antibody was applied. AEC (3-amino-9-ethylcarbazole) was used as chromogen. Subsequently, the slides were counterstained with hematoxylin for 1 minute, dehydrated in graded ethanol, and mounted in a conventional medium. The sections were examined under a photomicroscope (BX51 Olympus, Japan).

\section{Measurement of pro-inflammatory cytokine level in sciatic nerve}

The levels of tumor necrosis factor- $a(T N F-a)$ and interleukin$1 \beta(\mathrm{IL}-1 \beta)$ in the sciatic nerve specimens were measured to evaluate the inflammatory response induced by peripheral nerve injury. The Rat TNF-a ELISA Kit (Bendermed BMS 622) and Rat IL-1 ELISA Kit (BMS Bendermed 630) were used for measurements. The sciatic nerve was resected in the whole group after 30 days and stored at $-80^{\circ} \mathrm{C}$. The nerve samples were homogenized in $0.25 \mathrm{~mL}$ of ice-cold phosphate buffered saline and centrifuged. The levels of TNF- $a, I L-1 \beta$, and total protein in the supernatant were measured using the Lowry method with ELISA kits according to the manufacturer's instruction.

\section{Walking Track analysis and sciatic function index (SFI)}

Walking track analyses were performed in the animals at weeks 0 (after the surgery), 2, and 4 (before the sacrifice) using stamp and paper method (17). The lengths of the third toe to heel (PL), first to fifth toe (TS), and second toe to fourth toe (IT), on the experimental (E) and contralateral normal sides (N) were measured in all animals; sciatic function index (SFI) was calculated by using the following formula:

$\mathrm{SF}=-38.3 \times(\mathrm{EPL}-\mathrm{NPL}) / \mathrm{NPL}+109.5 \times(\mathrm{ETS}-\mathrm{NTS}) / \mathrm{NTS}+$ $13.3 \times(\mathrm{EIT}-\mathrm{NIT}) / \mathrm{NIT}-8.8$

SFI scores range from -100 (total dysfunction) to 0 (normal nerve function).

\section{Paw mechanical withdrawal threshold measurement}

Paw mechanical withdrawal threshold was measured using rat paw pressure analgesia-meter device (Ugo Basile, Italy), as described by Randall-Selitto (15). Quantitative measurement of pain was achieved by applying a mechanical force on the paws of the rats with a cone-shaped pusher with a rounded tip. The mechanical force (a maximum of $25 \mathrm{~g}$ ) was applied with increasing weight towards the dorsal surface of the hind paws of the rats which had underwent surgery, and then the weight was measured at the time when they pulled their paws. The withdrawal threshold of paws was measured four times and the average values used in the analysis. The measurements were transferred to the computer using SeDaCom v 2.0 software program. Responses to mechanical stimuli were measured before the surgical intervention and after 7,14 , and 30 days after the surgery.

\section{Statistical analysis}

Data pertaining to all variables are presented as mean \pm [Standard deviation (SD)]. SPSS version 15.0 was used to perform statistical analysis. Intergroup differences were 
Ozbek Z. et al: Effect of Genistein in Peripheral Nerve Injury

assessed by one-way Analysis of variance followed by Tukey's ad hoc test for multiple comparisons. $p$ values of $<0.05$ were considered indicative of a statistically significant intergroup difference.

\section{RESULTS}

\section{Immunohistochemical results}

GAP-43 and MBP immunoreactivity were similar in the sham and control groups. GAP-43 and MBP immunoreactivity in the genistein groups were higher than those in the other groups. GAP-43 and MBP immunoreactivity were higher in the gabapentin groups compared with that in the sham and control groups, but lower than that in the genistein groups (Figures $1 A-F$ and $2 A-F$ ). These results suggest that genistein improved nerve regeneration after traumatic nerve injury.

\section{Pro-inflammatory cytokine levels in sciatic nerve}

No significant different was observed between the genistein and gabapentin groups (crush+transection) with respect to the levels of IL-1 $\beta$ and TNF- $\alpha$ in the sciatic nerve samples $(p>0.05)$. There were statistically significant differences between the control and sham groups $(p<0.001)$ and among the control, genistein, and gabapentin groups $(p<0.001)$ (Figures 3,4$)$.

\section{Sciatic function index}

A statistically significant difference in functional recovery was observed among the control group, crush injury+genistein, and crush injury+gabapentin groups at $2^{\text {nd }}$ weeks $(p<0.01)$. No significant difference was observed in this respect between the control and transection groups $(p>0.05)$. However, at $4^{\text {th }}$ weeks, a statistically significant difference was observed between the control and all treatment groups (genistein and gabapentin) (Figure 5).

\section{Paw mechanical withdrawal threshold results}

Seven days after injury, the mechanical withdrawal threshold significantly decreased in comparison to that in the shamoperated animals $(p<0.001)$. On day 14 , a statistically significant difference was observed between the sham and control groups $(p<0.001)$ and between the sham and transection (+genistein, +gabapentin) groups $(p<0.05)$. No statistically significant differences were observed between the sham and crush injury (+genistein, +gabapentin) groups in this respect $(p>0.05)$. Further, no significant differences were observed between the sham and all treatment groups (genistein and gabapentin) on day 30 ( $p>0.05$ ); however, a significant difference was observed between the sham and control groups on day $30(p<0.001)$ (Figure 6).

\section{DISCUSSION}

Primary peripheral nerve injury results from compression, focal contusion, traction or transection of the nerve. This process is followed by secondary ischemic injury, which is dominated by inflammation (also referred to as degeneration).

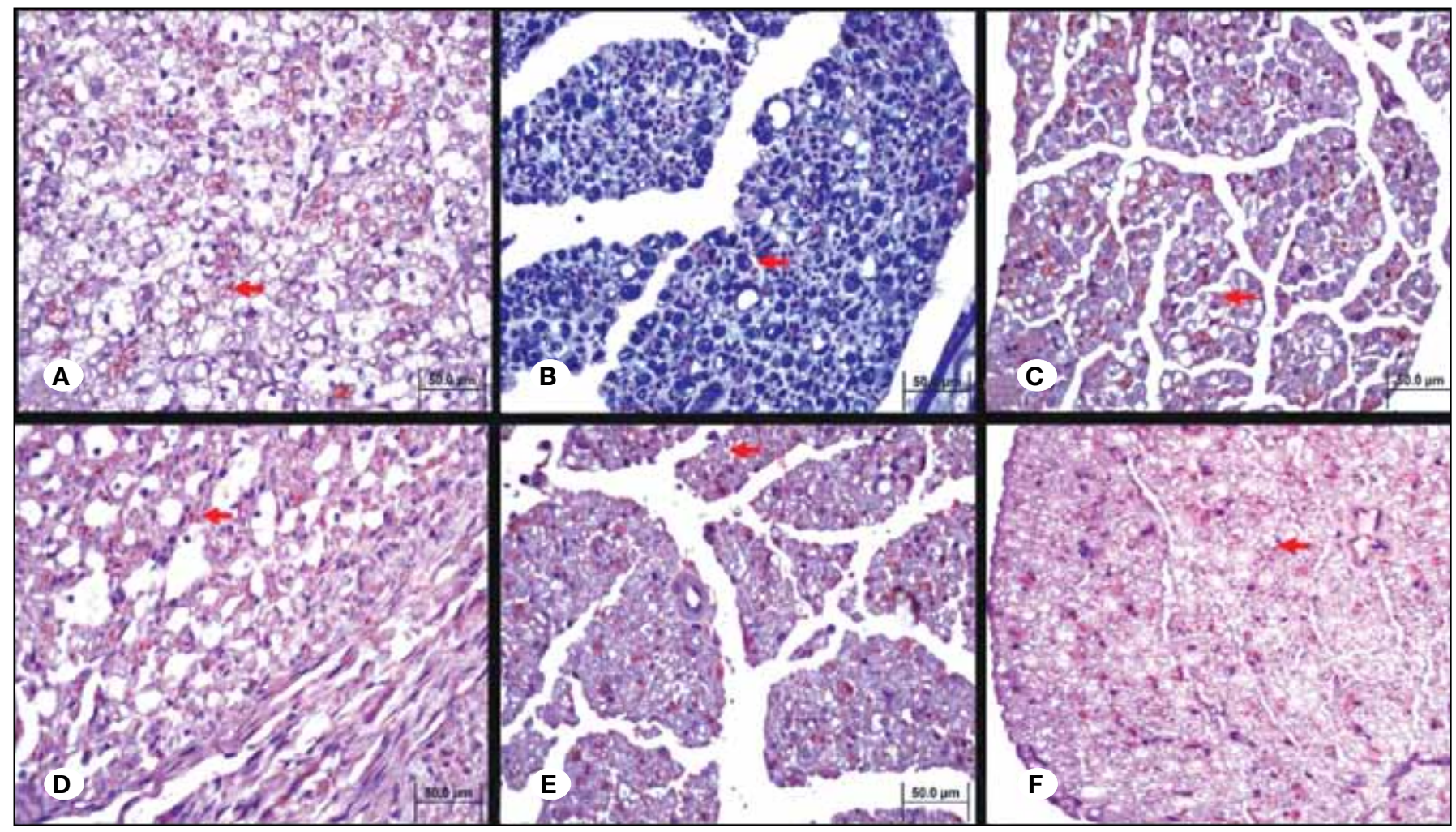

Figure 1: GAP-43 immunoreactivity. A) Sham, B) Control, C) Crush+Genistein, D) Crush+Gabapentin, E) Transection+Genistein, F)Transection+Gabapentin (GAP-43, x40). 
Cytokines, and in particular, TNF-a, play a major role in this inflammatory response. The degeneration is followed by regeneration and recovery. The axon grows by forming axonal colonies in the regeneration period and a new myelin sheath is formed. The important immunohistochemical markers of regeneration are GAP-43 and $\operatorname{MBP}(1,22)$. Motor functional improvement and reduction in neuropathic pain are also important indicators of neurological recovery after peripheral nerve injury.
In this study, the effects of genistein and gabapentin were compared after experimental peripheral nerve injury in rats. The results were examined on immunohistochemistry (GAP43, MBP), assessment of inflammatory cytokine expressions (IL-1 $\beta$, TNF- $\alpha$ ) and functional assessment (motor; SFI, neuropathic pain; paw withdrawal threshold). GAP-43 and MBP immunoreactivity in the genistein group was higher than that in the other groups on immunohistochemistry.

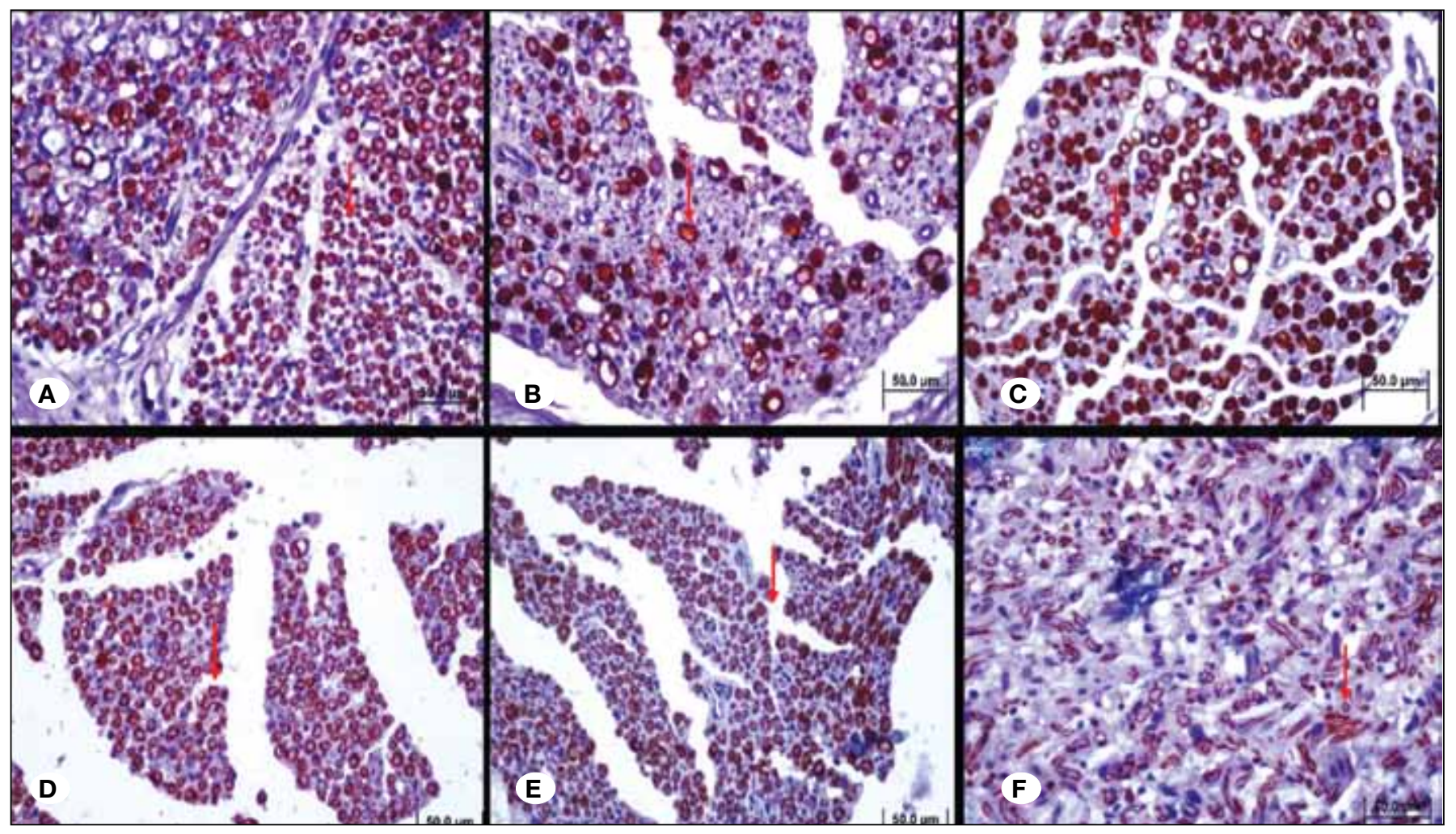

Figure 2: MBP immunoreactivity. A) Sham, B) Control, C) Crush+Genistein, D) Crush+Gabapentin, E) Transection+Genistein, F)Transection+Gabapentin (MBP, x40).

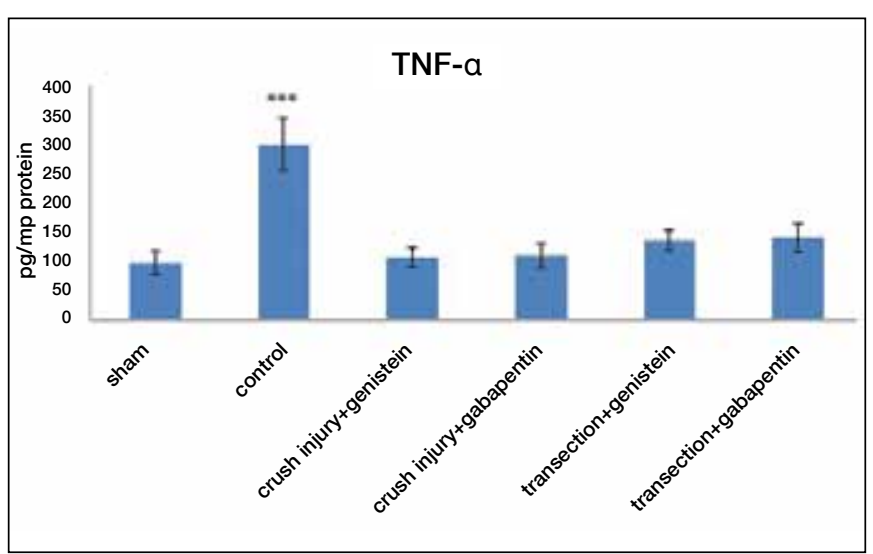

Figure 3: TNF-a levels. Bar graphs representing the TNF-a results as mean \pm SD. There was no statistically significant difference between sham, genistein and gabapentin groups ( $p>0.05)$. There were statistically significant differences between the control group and the other groups $\left({ }^{\star \star \star} \mathrm{p}<0.001\right)$.

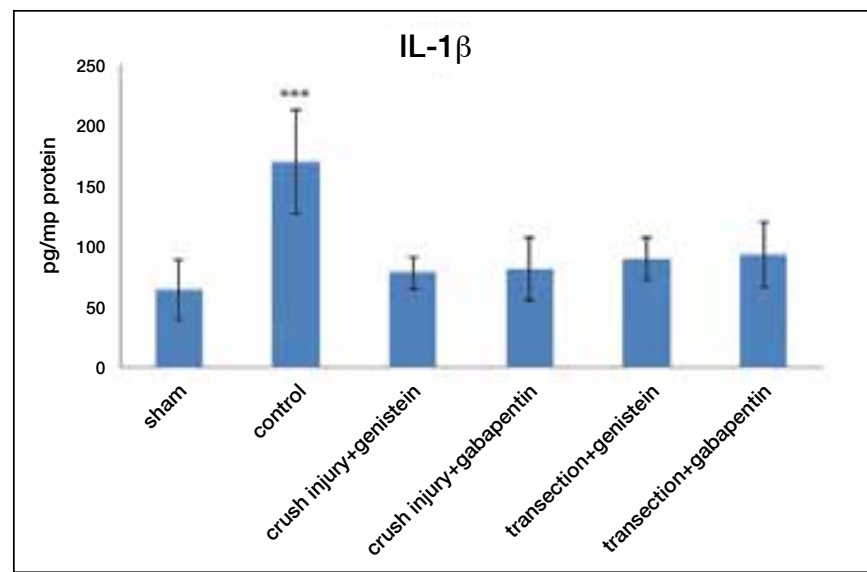

Figure 4: IL-1 $\beta$ levels. Bar graphs representing the IL-1 $\beta$ results as mean $\pm S D$. There was no statistically significant difference between sham, genistein and gabapentin groups $(p>0.05)$. There were statistically significant differences between the control group and the other groups $\left({ }^{\star \star *} p<0.001\right)$. 
With respect to the inflammatory and functional results, the effects of genistein and gabapentin were found to be comparable.

Genistein is a natural phytoestrogen isoflavone extracted from soybean (24). Its protective effect against various cancers is well documented (19). Its neuroprotective effects have also been demonstrated in in-vitro models of cerebral ischemia $(2,11)$. It was shown to remarkably reduce the neuropathic pain in diabetic peripheral neuropathy (24). A soy-rich diet was shown to attenuate hyperalgesia and allodynia after peripheral nerve injury (5), and these effects were attributed to genistein (23).

\section{SFI values}

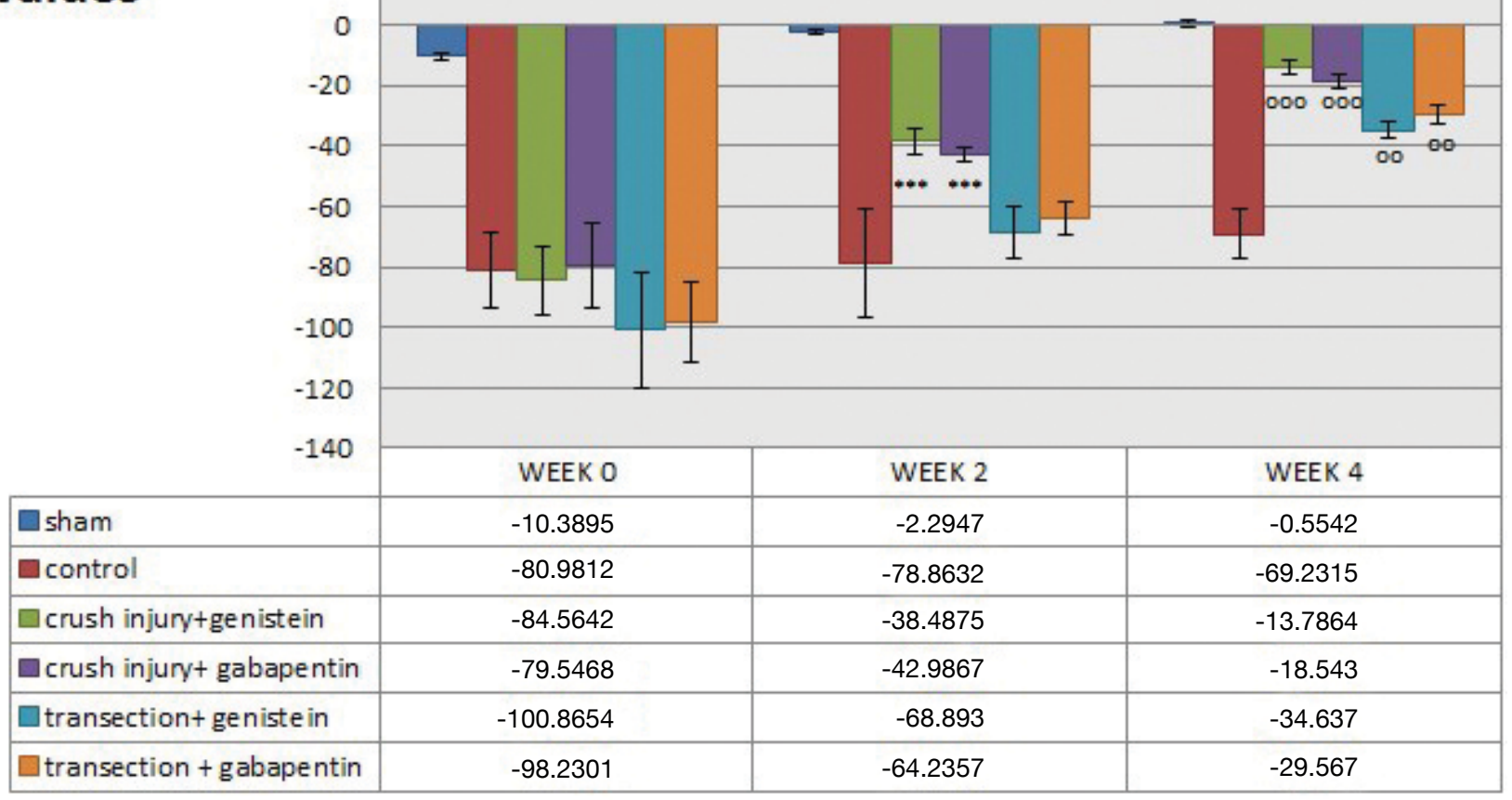

Figure 5: SFI values. Bar graphs representing the SFI values as mean $\pm S D$. ${ }^{* \star *} p<0.01$ versus control group at $2^{\text {nd }}$ week. ${ }^{000} p<0.0001$, oop $<0.001$ versus control group at $4^{\text {th }}$ week.

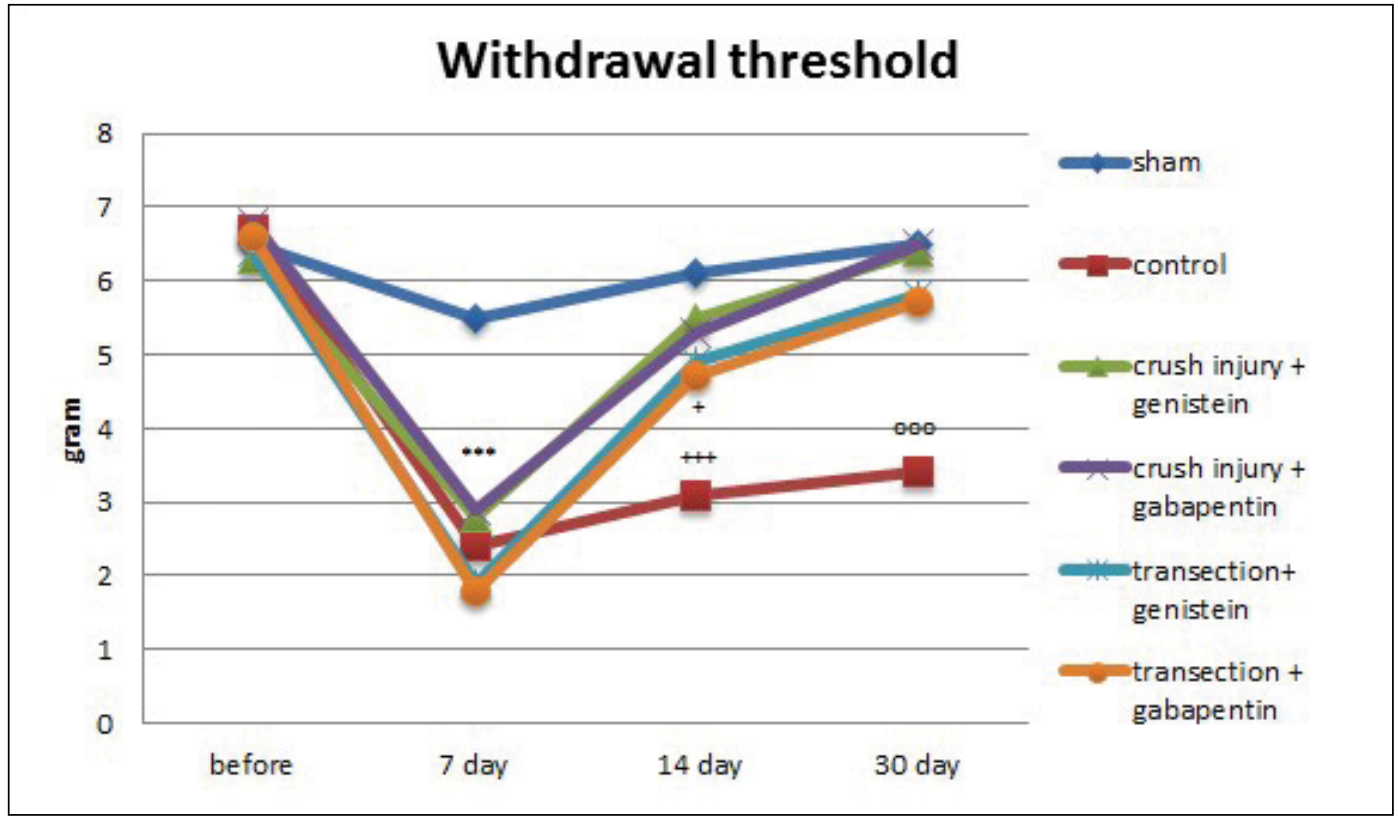

Figure 6: Mechanical withdrawal threshold results. ${ }^{\star \star *} p<0.001$ versus sham group at $7^{\text {th }}$ day. $+++p<0.001,+p<0.05$ versus sham group at $14^{\text {th }}$ day. ooop $<0.001$ versus sham group at $30^{\text {th }}$ day. 
Wallerian degeneration occurring after peripheral nerve injury is known to be regulated by pro-inflammatory cytokines (1214). The pathological cascade initiates at the site of injury and progresses to the dorsal root ganglia of the peripheral nerve. Subsequently, the glial cells and neurons participate in this cascade (14). Glial cells have been shown to activate microglial cells and astrocytes in the regional inflammation area (21). The most important cytokines of this cascade are TNF- $a$, IL-1 $\beta$, and IL-6 $(13,14)$. Of these, TNF- $\alpha$ is released by the schwann cells, mast cells, endothelial cells, and fibroblasts after injury and plays a key role in inducing upregulation of other inflammatory cytokines. Suppression of inflammatory cytokines after experimental peripheral nerve injury was shown to have a positive effect on neurological recovery $(3,4,9,24)$. The potent anti-inflammatory effect of gabapentin after sciatic nerve injury in rats has been demonstrated $(3,4,9)$. In our study, genistein and gabapentin showed a similar anti-inflammatory effect and reduced the level of cytokines in sciatic nerve tissue when compared with that in the control group.

In this study, the expressions of GAP-43 and MBP were measured to determine regeneration in the sciatic nerve injury. GAP-43 is a nervous tissue-specific structural protein which is present in the cytoplasm or on cell membrane in relation to the formation of axonal colonies and neuronal growth and plasticity $(1,22)$. It has a high expression level in nerve cells during regeneration and axonal growth. MBP is one of the important proteins which play a role in the myelination in the nerve cells. In the present study, we found a significant elevation in expression levels of GAP-43 and MBP in genistein and gabapentin treatment groups and MBP compared with that in the sham and control groups.

The SFI is a standard metric for the evaluation of motor function, which reflects nerve function after peripheral nerve injury (7). In our study, a significant motor deficit was seen in the crush injury and transection groups compared with that in the sham group in the early period. While the motor improvement was more obvious in the crush injury+genistein and crush injury+gabapentin groups compared with that in the control group at 2 weeks, no significant difference was observed between the control and transection groups. A significant motor improvement was observed in all groups except the control group at 4 weeks. The motor improvement occurred later in the transection (+genistein, +gabapentin) groups compared to the crush injury (+genistein, +gabapentin) groups.

In our study, the paw mechanical withdrawal threshold test was performed to assess the effect of genistein on neuropathic pain (24). The results were compared with those of gabapentin, which is known to attenuate neuropathic pain in previous animal experiments $(8,10)$. The pain threshold on day 7 was significantly lower in all groups compared with that in the sham group. On days 14 and 30 , the pain threshold was significantly higher in the crush injury (+genistein, +gabapentin) groups as compared to that in the control group. A significant improvement occurred later (on day 30 ) in the transection (+genistein, +gabapentin) groups. Our findings suggest that the increase in the neuropathic pain threshold was similar in the genistein and gabapentin groups.

\section{CONCLUSION}

Genistein promotes the neurological recovery after peripheral nerve injury. Our study results indicate that use of genistein as an alternative and complementary treatment for nerve injury should be encouraged.

\section{ACKNOWLEDGMENT}

This work was supported by Eskisehir Osmangazi University, Scientific Research Coordination Unit (Project Number: 2013358).

\section{- REFERENCES}

1. Benowitz LI, Routtenberg A: GAP-43: An intrinsic determinant of neuronal development and plasticity. Trends Neurosci 20(2):84-91, 1997

2. Burguete MC, Torregrosa G, Perez-Asensio FJ, CastelloRuiz M, Salom JB, Gil JV, Alborch E: Dietary phytoestrogens improve stroke outcome after transient focal cerebral ischemia in rats. Eur J Neurosci 23:703-710, 2006

3. Câmara CC, Araújo CV, de Sousa KK, Brito GA, Vale ML, Raposo Rda S, Mendonça FE, Mietto BS, Martinez AM, Oriá RB: Gabapentin attenuates neuropathic pain and improves nerve myelination after chronic sciatic constriction in rats. Neurosci Lett 607: 52-58, 2015

4. Câmara CC, Ramos HF, da Silva AP, Araújo CV, Gomes AS, Vale ML, Barbosa AL, Ribeiro RA, Brito GA, Costa CM, Oriá RB: Oral gabapentin treatment accentuates nerve and peripheral inflammatory responses following experimental nerve constriction in Wistar rats. Neurosci Lett 556:93-98, 2013

5. Campbell YJN, Raja SN, Seltzer Z: The correlation between dietary soy phytoestrogens and neuropathic pain behavior in rats after partial denervation. Anesth Analg 94:421-426, 2002

6. Cui SS, Yang CP, Bowen RC, Bai O, Li XM, Jiang W, Zhang X: Valproic acid enhances axonal regeneration and recovery of motor function after sciatic nerve axotomy in adult rats. Brain Res 975(1-2): 229-236, 2003

7. De Souza SA, Da Silva CA, Del Bel EA: Methodological evaluation to analyze functional recovery after sciatic nerve injury. J Neurotrauma 21(5): 627-663, 2004

8. Densmore VS, Kalous A, Keast JR, Osborne PB: Above-level mechanical hyperalgesia in rats develops after incomplete spinal cord injury but not after cord transection, and is reversed by amitriptyline, morphine and gabapentin. Pain 151(1):184-193, 2010

9. Dias JM, Brito TV, Magalhães AD, Silva Santos PW, Batista JA, Nascimento Dias EG, Fernandes BH, Damasceno SR, Silva RO, Aragão KS, Souza MH, Medeiros JV, Barbosa AL: Gabapentin, a synthetic analogue of gamma aminobutyric acid, reverses systemic acute inflammation and oxidative stress in mice. Inflammation 37(5):1826-1836, 2014

10. Gustafsson H, Flood K, Berge OG, Brodin E, Olgart L, Stiller CO: Gabapentin reverses mechanical allodynia induced by sciatic nerve ischemia and formalin-induced nociception in mice. Exp Neurol 182(2):427-434, 2003 
11. Liang HW, Qiu SF, Shen J, Sun LN, Wang JY, Bruce IC, Xia Q: Genistein attenuates oxidative stress and neuronal damage following transient global cerebral ischemia in rat hippocampus. Neurosci Lett 438:116-120, 2008

12. Martucci $C$, Trovato AE, Costa B: The purinergic antagonist PPADS reduces pain related behaviors and interleukin-1b, interleukin-6, iNOS and nNOS overproduction in central and peripheral nervous system after peripheral neuropathy in mice. Pain 137: 81-95, 2008

13. Moalem G, Tracey DJ: Immune and inflammatory mechanisms in neuropathic pain. Brain Res Rev 51(2):240-264, 2006

14. Myers RR, Campana WM, Shubayev VI: The role of neuroinflammation in neuropathic pain: Mechanisms and therapeutic targets. Drug Discov 11:8-20, 2006

15. Nogueira ES, Castro ER, Mancuso R, Navarro X: Randall Selitto test: A new approach for the detection of neuropathic pain after spinal cord injury. J Neurotrauma 29(5):898-904, 2012

16. Noorafshan A, Omidi A, Karbalay-Doust S: Curcumin protects the dorsal root ganglion and sciatic nerve after crush in rat. Pathol Res Pract 207(9): 577-582, 2011

17. Ozmen S, Ayhan S, Latifoglu O, Siemionow M: Stamp and paper method: A superior technique for the walking track analysis. Plast Reconstr Surg 109(5): 1760-1761, 2002

18. Panaite PA, Barakat-Walter I: Thyroid hormone enhances transected axonal regeneration and muscle reinnervation following rat sciatic nerve injury. J Neurosci Res 88(8):17511763, 2010
19. Qian Y, Guan T, Huang M, Cao L, Li Y, Cheng H, Jin H, Yu D: Neuroprotection by the soy isoflavone, genistein, via inhibition of mitochondria dependent apoptosis pathways and reactive oxygen induced-NF-kappa B activation in a cerebral ischemia mouse model. Neurochem Int 60:759-767, 2012

20. Schenker M, Riederer BM, Kuntzer T, Barakat-Walter I: Thyroid hormones stimulate expression and modification of cytoskeletal protein during rat sciatic nerve regeneration. Brain Res 957(2):259-270, 2002

21. Scholz J, Woolf CJ: The neuropathic pain triad: Neurons, immune cells and glia. Nat Neurosci 10:1361-1368, 2007

22. Skene JHP: Axonal growth-associated proteins. Annu Rev Neurosci 12:127-156, 1989

23. Valsecchi $A E$, Franchi $S$, Panerai $A E$, Rossi $A$, Sacerdote $P$, Colleoni M: The soy isoflavone genistein reverses oxidative and inflammatory state, neuropathic pain, neurotrophic and vasculature deficits in diabetes mouse model. Eur J Pharmacol 650(2-3): 694-702, 2011

24. Valsecchi AE, Franchi S, Panerai AE, Sacerdote P, Trovato $A E$, Colleoni $M$ : Genistein, a natural phytoestrogen from soy, relieves neuropathic pain following chronic constriction sciatic nerve injury in mice: Anti-inflammatory and antioxidant activity. J Neurochem 107(1): 230-240, 2008

25. Wu F, Xing D, Peng Z, Rao T: Enhanced rat sciatic nerve regeneration through silicon tubes implanted with valproic acid. J Reconstr Microsurg 24(4): 267-276, 2008 